\title{
CUANTIFICACIÓN DEL POTENCIAL EMPRENDEDOR DE LOS ALUMNOS DE LA FACULTAD DE CIENCIAS ADMINISTRATIVAS DE LA UNIVERSIDAD NACIONAL MAYOR DE SAN MARCOS
}

\author{
Emma Pérez Palacios* \\ eeperezpalacios@yahoo.com \\ Justa Caridad Huaroto Sumari de Sambrano*** \\ jhuarotos@yahoo.com \\ Gabriela Montes Quintana de Domínguez ${ }^{* * * * *}$ \\ gmontesq@unmsm.edu.pe \\ José Luis Uyehara Benites ${ }^{* * * * * * *}$ \\ uyehrarapp58@yahoo.es
}

\begin{abstract}
RESUMEN
Una de las opciones de realización personal y profesional de los estudiantes universitarios es crear una empresa, hacerla crecer, generar empleo y contribuir al desarrollo local y nacional. Pero, ¿cuán desarrolladas están las capacidades emprendedoras de los jóvenes universitarios?. La presente investigación no experimental intenta determinar el potencial emprendedor utilizando la metodología cuantitativa, deductiva, y básica con diseño transversal y correlacional en una poblacional total de 396 estudiantes de las tres escuelas (Turismo, Administración y Negocios Internacionales, ciclos: III, V, y VII) de la Facultad de Ciencias Administrativas. Utilizando la encuesta como técnica de recolección de dato y como instrumento, se aplicó el Test del Emprendedor desarrollado por el Ministerio de Trabajo y Promoción del Empleo, el mismo que cuenta con las validaciones de rigor científico. En el tratamiento estadístico se empleó el procesador estadístico IBM-SPSS versión 20.01 para asignación de puntajes, tabulaciones, gráficos e indicadores estadísticos. Los resultados de la investigación determinaron lo siguiente: la característica Iniciativa y Visionario son las que obtienen los puntajes más altos en los alumnos de la Facultad de Ciencias Administrativas. Los alumnos de los primeros ciclos evidencian los puntajes más bajos, esto significa que ingresan a la universidad sin una expectativa respecto a la creación de empresas. Finalmente, se evidencia de acuerdo a los resultados de la Prueba Estadística
\end{abstract}

Licenciada en Estadística. Magister en Administración. Maestría en Métodos Cuantitativos de la Economía. Post grado: Docencia Universitaria y Proyectos de inversión. Experiencia Laboral: INEI, CONASEV, OSIPTEL, APOYO Opinión y Mercado, CIP. Docente Universitario: UNMSM, ULima, UCV, UFV, UNI.

** Licenciada en Estadística, Maestría en Estadística Matemática, por la UNMSM. Maestría en Métodos Cuantitativos de la Economía, por la UNMSM. Experiencia laboral: Docente Asociada del Departamento Académico de Estadística, de la Facultad de Ciencias Matemática, de la UNMSM. Universidad de Lima.

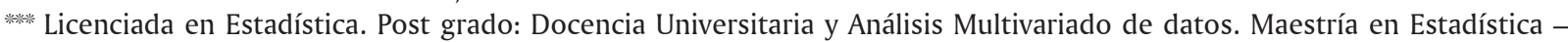
Experiencia Laboral: INEI y Empresa de Estudios de Mercado Alegre y Asociados. Docente Asociada del Departamento Académico de Estadística, de la Facultad de Ciencias Matemática. Universidad de Lima.

******:Licenciado en Psicología. Post grado en Neuro Psicología Infantil y en Medicina Tradicional Coreana Acupuntura y Moxibustión. Experiencia Laboral: Psicólogo asimilado a la Sanidad de las Fuerzas Policiales, Jefe del Departamento de Psicología de la Aldea Infantil WestafliaKinderdorfFilial.Perú, Instituto Nacional de Medicina Tradicional Peruana Docente Universitario: UCV, Universidad Enrique Guzmán y Valle.- La Cantuta, Universidad Particular Norbert Wiener. Ponente de Conferencias: Derrama Magisterial y Gobiernos Regionales. 
Chi Cuadrado que existe un comportamiento recurrente y homogéneo respecto a las características emprendedoras en las tres escuelas de la Facultad de Ciencias Administrativas de la Universidad Nacional Mayor de San Marcos, así como a nivel los ciclos académicos iniciales y avanzados.

Palabras clave: Emprendimiento, Características Emprendedoras, Test del Emprendedor.

\begin{abstract}
SUMMARY
One option of personal and professional development of students is to start a business, make it grow, create jobs and contribute to local and national development. But, How developed are the entrepreneurial skills of university students?

This experimental investigation attempts to determine the ENTREPRENEURIAL POTENTIAL using deductive, and basic quantitative methodology, with correlational cross-sectional design in a total population of 396 students from Faculty of Administrative Sciences. (Tourism, Management and International Business students from Third, fifth and seventh semester)

Using the survey as data collection technique and as the instrument of the Entrepreneur Test this is developed by the Ministry of Labour and Employment Promotion, which has the same scientific rigor validations.

In the statistical treatment was used statistical processor IBM-SPSS version 20.01 for assigning scores, tabs, charts and statistical indicators.

The results of the investigation determined the following: Initiative and Visionary feature are those of the highest scores in the students of the Faculty of Administrative Sciences, students in the first few semesters show lower scores, this means that they enter in college without expectations regarding entrepreneurship.

Finally, the evidence according to the results of the Chi Square test statistic that there is a recurring and consistent behavior regarding the entrepreneurial characteristics in Tourism, Management and International Business students of the faculty of Administrative Sciences of Universidad Mayor de San Marcos, as well as at the initial and advanced academic semesters.
\end{abstract}

Keywords: Entrepreneurship, Entrepreneurial Characteristics, Entrepreneurial Test.

\section{INTRODUCCIÓN}

Podemos considerar que la primera idea de emprender un negocio propio nace cuando los individuos sienten que sus esfuerzos en el trabajo no son suficientemente recompensados, o cuando pasan a formar parte del grueso de desempleados del país. El crecimiento del desempleo ha causado que más y más personas hayan optado por crear sus propias fuentes de ingreso con el propósito de mejorar su nivel de vida. Desde sus experiencias vividas como trabajadores dependientes, es posible que consideren tener los contactos suficientes y los recursos financieros necesarios para lanzarse a la actividad empresarial pues esto supone tener mayor libertad y seguridad, ser prestigioso al ser dueño de una empresa y tener control sobre cuántas horas trabajar, a quién contratar, entre otros; sin embargo a pesar de que estas son numerosas ventajas, también se presentan obstáculos como los aspectos administrativos y financieros, acceso a fuentes de información, burocracia, etc.

Llevar adelante un emprendimiento propio requiere una amplia gama de habilidades y un conjunto de atributos de personalidad; por ello, antes de lanzarse a la actividad empresarial, hay que asegurarse de poseer un perfil emprendedor $y$ de no tenerlo, es posible que pueda desarrollarlo.

La principal barrera al emprender es precisamente la escasa o nula preparación empresarial, entendiendo por esta preparación el desarrollo de capacidades y habilidades; entonces, vale la pena preguntarse si se cuenta con las características necesarias para ser un emprendedor; es muy importante hacerlo más temprano que tarde, pues de esta forma la persona será consciente de sus reales posibilidades para alcanzar el éxito. Es mejor descubrir ahora qué cualidades necesita antes de que sea demasiado tarde.

\section{MÉTODOS}

\section{Características del estudio}

El estudio se basa en evaluar la Aptitud de Emprendimiento de una poblacional total de 396 estudiantes de las tres Escuelas (Turismo, Administración, Negocios Internacionales, ciclos: III, V, y VII) de la Facultad de Ciencias Administrativas de la Universidad Nacional Mayor de San Marcos (UNMSM). Dicha encuesta se aplicó en el primer periodo del año 2012. 


\section{Métodos, técnicas e instrumentos}

Los métodos de investigación fueron el Cuantitativo, Descriptivo, Hipotético-Deductivo y Estadístico, Cuantitativo por la metodología general; Descriptivo porque permite describir las variables en estudio estableciendo relaciones; Hipotético - deductivo, porque determina la comprobación de las hipótesis y Estadístico, porque el contraste de las hipótesis se rigen por la teoría de la probabilidad.

Instrumento Test del Emprendedor, desarrollado en el Ministerio de Trabajo, explorándose las siguientes dimensiones o características emprendedoras:

- Visionario.

- Tolerante a la incertidumbre.

- $\quad$ Líder.

- Responsable.

- Perseverante.

- Optimista.

- Posee iniciativa.

- Administra óptimamente sus recursos vitales: tiempo y dinero.
- Confía en sí mismo y en los demás.

- Tiene deseos de superación e independencia.

- Tiene capacidad para crear e innovar.

Los resultados obtenidos describen las características emprendedoras de los alumnos, diferenciándose por escuela y por ciclo de estudios. Los puntajes se clasifican en tres categorías:

- Bajo nivel de emprendimiento.

- Medio nivel de emprendimiento (promedio).

- Alto nivel de emprendimiento.

\section{RESULTADOS}

Estadígrafos del puntaje total y de las características emprendedoras.

Los estudiantes de la Facultad de Ciencias Administrativas de la UNMSM presentan puntajes promedio en la puntuación total y la mayoría de las características emprendedoras, a excepción de la característica Responsable y confianza que están categorizadas como bajas.

Sin embargo los mayores puntajes se encuentran en las características: Administra su tiempo, Tolerante y Líder.

Cuadro $\mathrm{N}^{\circ} 1$ Estadígrafos del puntaje total y de las características emprendedoras

\begin{tabular}{|c|c|c|c|c|c|c|c|}
\hline & & & & & & \\
\hline & CARACTERISETICA & Media & Categoría & Desv.Est. & C.V & Mínimo & Máximo \\
\hline 1 & VISIONARIO & 9.8 & Promedio & 2.1 & 21.7 & 2 & 12 \\
\hline 2 & TOLERANTE & 11.2 & Promedio & 2.3 & 20.3 & 4 & 15 \\
\hline 3 & LIDER & 11.3 & Promedio & 2.1 & 18.4 & 5 & 16 \\
\hline 4 & RESPONSABLE & 8.6 & Bajo & 2.3 & 26.6 & 0 & 12 \\
\hline 5 & PERSEVERANTE & 7.6 & Promedio & 1.5 & 19.9 & 0 & 9 \\
\hline 6 & OPTIMISTA & 4.5 & Promedio & 1.1 & 24.2 & 0 & 6 \\
\hline 7 & ADMINISTRA & 11.5 & Promedio & 2.5 & 22.0 & 2 & 18 \\
\hline 8 & CONFÍA & 5.7 & Bajo & 1.4 & 25.5 & 1 & 9 \\
\hline 9 & SUPERACIÓN & 4.0 & Promedio & 1.6 & 39.0 & 0 & 6 \\
\hline 10 & CREATIVO & 6.7 & Promedio & 1.5 & 22.8 & 0 & 9 \\
\hline 11 & INICIATIVA & 7.3 & Promedio & 1.8 & 24.3 & 2 & 9 \\
\hline 12 & PUNTAJE TOTAL & 88.1 & Promedio & 10.8 & 12.2 & 56 & 108 \\
\hline
\end{tabular}


Gráfico N. ${ }^{\circ} 1$ Categoría de Empredimiento

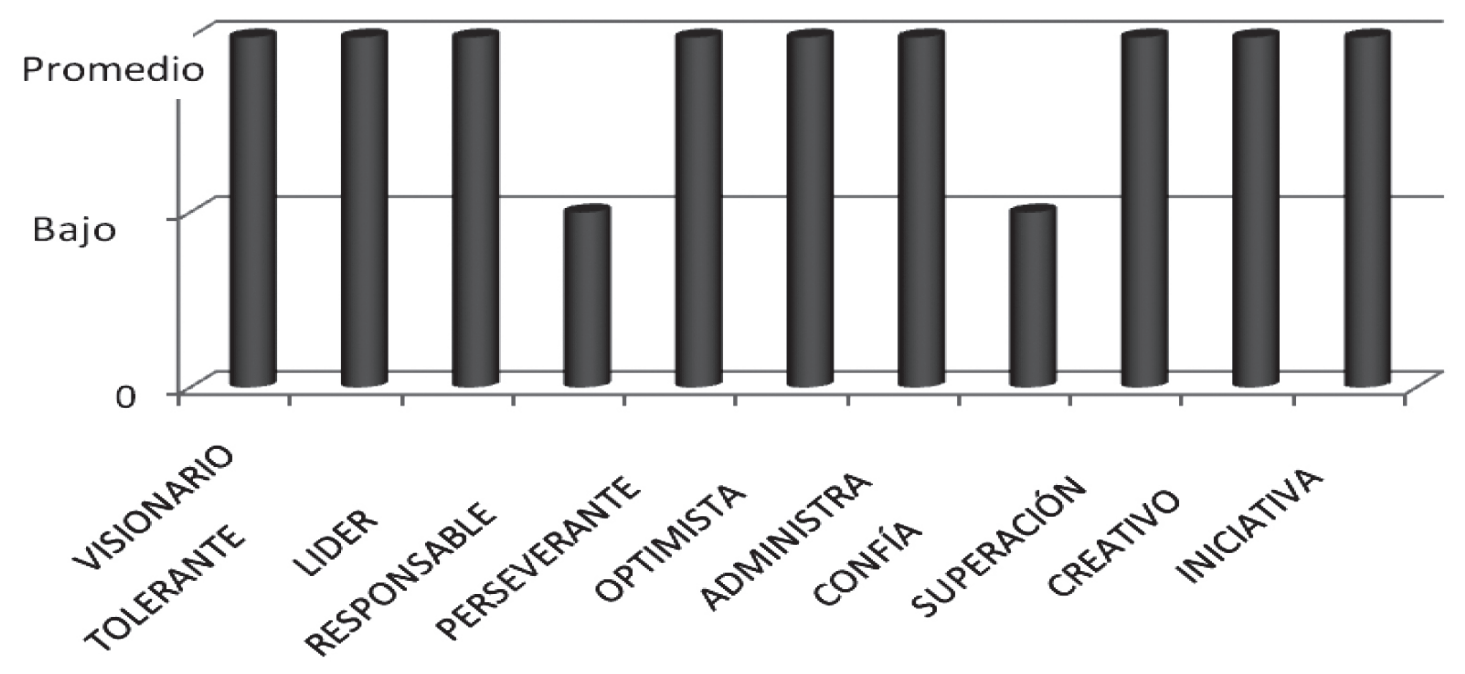

En cuanto a la distribución nivel de emprendimiento entre las características se puede apreciar lo siguiente: hay mayor porcentaje de alumnos con puntajes altos en las características Visionario y con Iniciativa y con los más bajos Superación y Confianza.
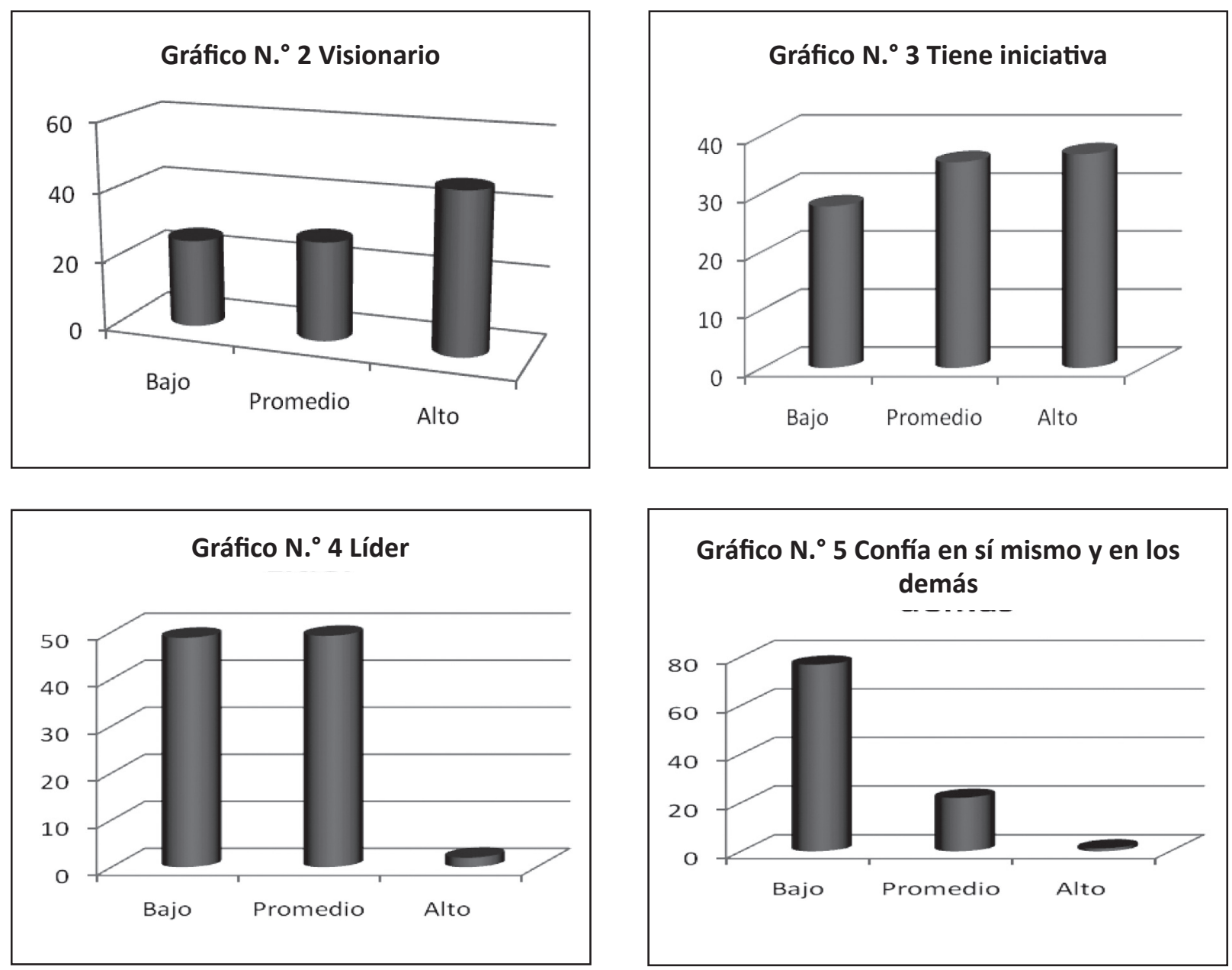

Gestión en el Tercer Milenio, Revista de Investigación de la Fac. de CC. Administrativas, UNMSM (Vol. 16, N. ${ }^{\circ}$ 32, Lima, Diciembre de 2013) 
Cuadro $\mathrm{N}^{\circ} 2$ Visionario

\begin{tabular}{|l|c|c|}
\hline Niveles & Conteo & Porcentaje \\
\hline Bajo & 100 & 25,3 \\
\hline Promedio & 113 & 28,5 \\
\hline Alto & 183 & 46,2 \\
\hline Total & 396 & 100,0 \\
\hline
\end{tabular}

Cuadro N. ${ }^{\circ} 3$ Tolerante

\begin{tabular}{|l|c|c|}
\hline Niveles & Conteo & Porcentaje \\
\hline Bajo & 90 & 22,7 \\
\hline Promedio & 242 & 61,1 \\
\hline Alto & 64 & 16,2 \\
\hline Total & 396 & 100,0 \\
\hline
\end{tabular}

Cuadro N. ${ }^{\circ} 4$ Líder

\begin{tabular}{|l|c|c|}
\hline Niveles & Conteo & Porcentaje \\
\hline Bajo & 193 & 48,8 \\
\hline Promedio & 195 & 49,2 \\
\hline Alto & 8 & 2,0 \\
\hline Total & 396 & 100,0 \\
\hline
\end{tabular}

Cuadro N. ${ }^{\circ} 5$ Responsable

\begin{tabular}{|l|c|c|}
\hline Niveles & Conteo & Porcentaje \\
\hline Bajo & 176 & 44,4 \\
\hline Promedio & 208 & 52,6 \\
\hline Alto & 12 & 3,0 \\
\hline Total & 396 & 100,0 \\
\hline
\end{tabular}

Cuadro N. ${ }^{\circ} 6$ Perseverante

\begin{tabular}{|l|c|c|}
\hline Niveles & Conteo & Porcentaje \\
\hline Bajo & 2 & 5,1 \\
\hline Promedio & 265 & 66,9 \\
\hline Alto & 111 & 28,0 \\
\hline Total & 378 & 100,0 \\
\hline
\end{tabular}

Cuadro N. ${ }^{\circ} 7$ Optimista

\begin{tabular}{|l|c|c|}
\hline Niveles & Conteo & Porcentaje \\
\hline Bajo & 59 & 14,9 \\
\hline Promedio & 178 & 45,0 \\
\hline Alto & 159 & 40,1 \\
\hline Total & 396 & 100,0 \\
\hline
\end{tabular}

Cuadro $\mathrm{N}^{\circ} 8$ Administra su tiempo

\begin{tabular}{|l|c|c|}
\hline Niveles & Conteo & Porcentaje \\
\hline Bajo & 127 & 32,1 \\
\hline Promedio & 251 & 63,4 \\
\hline Alto & 18 & 4,5 \\
\hline Total & 396 & 100,0 \\
\hline
\end{tabular}

Cuadro N. ${ }^{\circ} 9$ Confía

\begin{tabular}{|l|c|c|}
\hline Niveles & Conteo & Porcentaje \\
\hline Bajo & 304 & 76,8 \\
\hline Promedio & 87 & 22 \\
\hline Alto & 5 & 1,2 \\
\hline Total & 396 & 100,0 \\
\hline
\end{tabular}

Cuadro N..$^{\circ} 10$ Superación

\begin{tabular}{|l|c|c|}
\hline Niveles & Conteo & Porcentaje \\
\hline Bajo & 231 & 58,4 \\
\hline Promedio & 88 & 22,2 \\
\hline Alto & 77 & 19,4 \\
\hline Total & 396 & 100,0 \\
\hline
\end{tabular}

Cuadro N. ${ }^{\circ} 11$ Creativo

\begin{tabular}{|l|c|c|}
\hline Niveles & Conteo & Porcentaje \\
\hline Bajo & 74 & 18,7 \\
\hline Promedio & 279 & 70,5 \\
\hline Alto & 43 & 10,9 \\
\hline Total & 396 & 100,1 \\
\hline
\end{tabular}

Cuadro $\mathrm{N}^{\circ}{ }^{\circ} 12$ Tiene iniciativa

\begin{tabular}{|l|c|c|}
\hline Niveles & Conteo & Porcentaje \\
\hline Bajo & 110 & 27,8 \\
\hline Promedio & 140 & 35,4 \\
\hline Alto & 146 & 36,8 \\
\hline Total & 396 & 100,0 \\
\hline
\end{tabular}

Cuadro N..$^{\circ} 13$ General

\begin{tabular}{|l|c|c|}
\hline Niveles & Conteo & Porcentaje \\
\hline Bajo & 48 & 12,1 \\
\hline Promedio & 347 & 87,6 \\
\hline Alto & 1 & 0,3 \\
\hline Total & 396 & 100,0 \\
\hline
\end{tabular}


Estadígrafos del puntaje total y de las características emprendedoras por Escuela Profesional de la Facultad de Ciencias Administrativas de la UNMSM

No se encuentra evidencia de diferencia significativa en los puntajes por características emprendedoras y las Escuelas Profesionales, salvo en la característica Optimista. Sin embargo, la diferencia no es considerada abrumadora.
En la distribución porcentual de los estudiantes de la muestra de acuerdo a las escuelas profesionales y puntaje total. Se puede apreciar que el nivel de emprendimiento entre las escuelas profesionales predominante es el promedio. No se aprecia diferencias significativas entre las carreras profesionales. De acuerdo a la prueba Chi Cuadrado se acepta la hipótesis nula de la igualdad de distribución de los puntajes en las tres escuelas.

Cuadro N. ${ }^{\circ} 14$ Puntaje Total

\begin{tabular}{|c|c|c|c|c|}
\hline & Bajo & Promedio & Alto & Total \\
\hline Administrac. & 5.3 & 35.4 & 0.3 & 40.9 \\
\hline NN.II. & 3.0 & 29.6 & 0.0 & 32.6 \\
\hline Turismo & 3.8 & 22.7 & 0.0 & 26.5 \\
\hline Total & 12.1 & 87.6 & 0.3 & 100.0 \\
\hline
\end{tabular}

Gráfico N. ${ }^{\circ} 6$ Puntaje Total

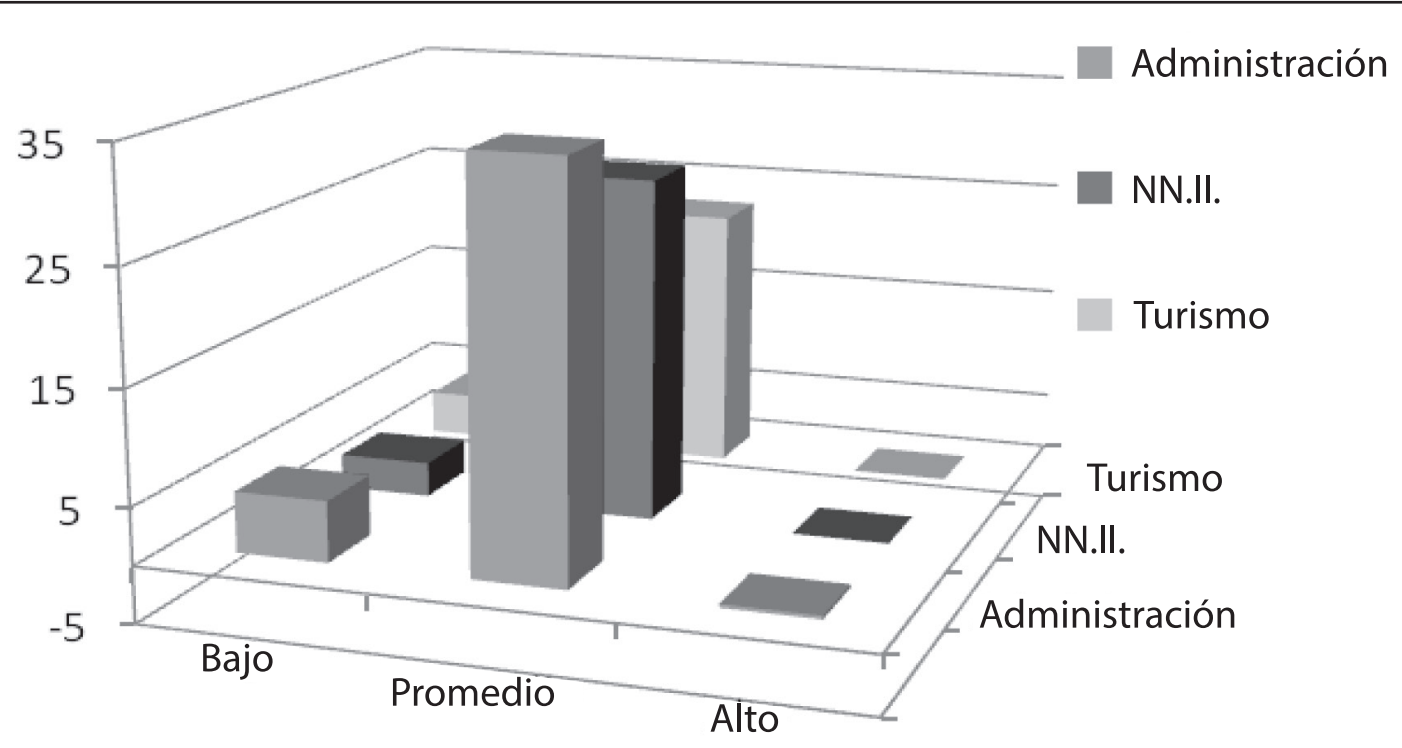


- Resultados de la Prueba Chi cuadrado: Para comparar el Nivel de emprendimiento y las Escuelas Profesionales

Los conteos esperados se imprimen debajo de los conteos observados Las contribuciones Chi-cuadradas se imprimen debajo de los conteos esperados

\section{Cuadro N. ${ }^{\circ} 15$ Resultados de Prueba Chi-Cuadrado}

\begin{tabular}{|c|c|c|c|}
\hline & Ваjo & Promedio & Total \\
\hline \multirow[t]{3}{*}{ Adm } & 22 & 140 & 162 \\
\hline & 20,05 & 141,95 & \\
\hline & 0,191 & 0,027 & \\
\hline \multirow[t]{3}{*}{$\begin{array}{c}\text { Negocios } \\
\text { internacionales }\end{array}$} & 12 & 117 & 129 \\
\hline & 15,96 & 113,04 & \\
\hline & 0,983 & 0,139 & \\
\hline \multirow[t]{3}{*}{ Turism } & 15 & 90 & 105 \\
\hline & 12,99 & 92,01 & \\
\hline & 0,310 & 0,044 & \\
\hline Total & 49 & 347 & 396 \\
\hline
\end{tabular}

Ho: Las Escuelas Profesionales tienen la misma distribución del nivel de emprendimiento.

H1: Las Escuelas Profesionales tienen diferente distribución del nivel de emprendimiento.

En la distribución Porcentual de los estudiantes de la muestra de acuerdo a los niveles de estudio (ciclos) y los niveles de emprendimiento del puntaje total, tenemos que de acuerdo a los puntajes la mayoría de las características emprendedoras, tienen una calificación catalogada como promedio, sin existir diferencia entre los niveles de estudio, salvo en las características Optimista y Responsable.

En términos generales se puede apreciar que el nivel de emprendimiento entre niveles de estudio (ciclos) es el promedio.

Cuadro $\mathrm{N}^{\circ} 16$ Niveles de empredimiento

\begin{tabular}{|l|c|c|c|c|}
\hline & Bajo & Promedio & Alto & Total \\
\hline Avanzados & 2,5 & 38,4 & 0,0 & 40,9 \\
\hline Iniciales & 9,6 & 49,2 & 0,3 & 59,1 \\
\hline Total & 12,1 & 87,6 & 0,3 & 100,0 \\
\hline
\end{tabular}

- Resultados de la Prueba Chi cuadrado para comparar el nivel de emprendimiento y los ciclos de estudio

Hay relación entre los ciclos académicos iniciales y avanzados y el nivel de emprendimiento bajo o promedio. A mayor avance académico mejor nivel de emprendedorismo.

\section{Cuadro N. ${ }^{\circ} 15$ Resultados de Prueba Chi-Cuadrado}

$\begin{array}{cccc} & \text { Bajo } & \text { Promedio } & \begin{array}{c}\text { Total pro- } \\ \text { medio }\end{array} \\ \text { Iniciales } & 39 & 195 & 234 \\ & 28,95 & 205,05 & \\ & 3,485 & 0,492 & \\ \text { Avanzados } & 10 & 152 & 162 \\ & 20,05 & 141,95 & \\ \text { Total } & 5,034 & 0,711 & \\ & 49 & 347 & 396 \\ \text { Chi-cuadrada } & =9,722, \mathrm{GL}=1, \text { Valor } \mathrm{P}= \\ \text { 0,002 } & & & \end{array}$

Ho: Los niveles de estudios tienen la misma distribución del nivel de emprendimiento.

H1: Los niveles de estudios diferente distribución del nivel de emprendimiento.

Como el p-valor $0,002<0,05$ Ho se rechaza y se acepta $\mathrm{H} 1$.

En las únicas características en las cuales se observaron diferencias significativas entre los niveles de emprendimiento y los ciclos de estudios son:

Cuadro N. ${ }^{\circ} 18$ Tolerante: niveles de emprendimiento

\begin{tabular}{|l|c|c|c|c|}
\hline Niv. Estudios & Bajo & Promedio & Alto & Total \\
\hline Ciclos iniciales & 16,7 & 35,4 & 7,0 & 59,1 \\
\hline Ciclos avanzados & 6,1 & 25,8 & 9,0 & 40,9 \\
\hline Total & 22,8 & 61,2 & 16,0 & 100,0 \\
\hline
\end{tabular}

Cuadro N. ${ }^{\circ} 19$ Responsable: niveles de emprendimiento

\begin{tabular}{|l|c|c|c|c|}
\hline Niv. Estudios & Bajo & Promedio & Alto & Total \\
\hline Ciclos iniciales & 30,8 & 27,3 & 1,0 & 59,1 \\
\hline Ciclos avanzados & 13,6 & 25,3 & 2,0 & 40,9 \\
\hline Total & 44,4 & 52,6 & 3,0 & 100,0 \\
\hline
\end{tabular}


Cuadro N. $^{\circ} 20$ Administra su tiempo: niveles de emprendimiento

\begin{tabular}{|l|c|c|c|c|}
\hline Niv. Estudios & Bajo & Promedio & Alto & Total \\
\hline Ciclos iniciales & 21,7 & 35,6 & 1,8 & 59,1 \\
\hline Ciclos avanzados & 10,3 & 27,8 & 2,8 & 40,9 \\
\hline Total & 32 & 63,4 & 4,6 & 100,0 \\
\hline
\end{tabular}

Cuadro N. ${ }^{\circ} 21$ Superación: niveles de emprendimiento

\begin{tabular}{|l|c|c|c|c|}
\hline Niv. Estudios & Bajo & Promedio & Alto & Total \\
\hline Ciclos iniciales & 36.8 & 9.7 & 12.5 & 59 \\
\hline Ciclos avanzados & 21.5 & 12.4 & 7.1 & 41 \\
\hline Total & 58.3 & 22.1 & 19.6 & 100 \\
\hline
\end{tabular}

Cuadro N. ${ }^{\circ} 22$ Creatividad: niveles de emprendimiento

\begin{tabular}{|l|c|c|c|c|}
\hline Niv. Estudios & Bajo & Promedio & Alto & Total \\
\hline Ciclos iniciales & 12.6 & 42.0 & 4.5 & 59.1 \\
\hline Ciclos avanzados & 6.1 & 28.5 & 6.3 & 40.9 \\
\hline Total & 18.7 & 70.5 & 10.8 & 100 \\
\hline
\end{tabular}

\section{Discusión}

Es preocupante que los estudiantes de la Facultad de Ciencias Administrativas de las tres Escuelas Profesionales: Administración, Negocios Internacionales y Turismo de la Universidad Mayor de San Marcos, NO lograron obtener puntajes en sus características en la categoría de nivel alto. Se observa, de igual manera, puntajes insuficientes que lo clasifican en la categoría de nivel bajo en tres características del Test del Emprendimiento que son: Confianza en sí mismo y en los demás, Superación y Responsable. Otro análisis deductivo preocupante es la NO existencia de diferencias altamente significativas entre las características del Test del Emprendimiento en los estudiantes de los primeros ciclos y los avanzados, esto es una señal de alerta y de reflexión ya que la influencia de la vida universitaria en sus espectros multidimensionales es insuficiente o poco motivador a en la formación de talentos, gestores y líderes profesionales. Por ello es importante replantear nuestros esquemas y paradigmas educativos. Dentro de este contexto se deben de diseñar y desarrollar programas al corto y largo plazo enfocados en elevar el nivel de emprendimiento de la categoría promedio a la de categoría nivel alto.

Para lograr dicho fin se deben desarrollar programas de sensibilización de formación y capacitación en tres ejes de desarrollo integral, desarrollo personal (Autoestima, Asertividad, Asertividad, Liderazgo, Inteligencia emocional, Empatía, Comunicación, Creatividad, etc.) el desarrollo humano (valores, ética, etc.) y la salud físico-mental. Estas programaciones enfocadas en estos tres ejes de desarrollo a su vez serán diferenciados por el perfil que tengan cada escuela profesional de acuerdo a cada objetivo.

Dentro de este marco conceptual debe llevarse a cabo la ejecución de proyectos de gestión y de productividad, articulando la teoría y la práctica de la formación profesional con el emprendimiento, estimulando las habilidades, destrezas, capacidades y competencias hacia la excelencia y la competitividad.

La universidad tiene la responsabilidad de contribuir al emprendimiento de los estudiantes en su proceso de desarrollo personal, profesional, social y de valores como parte de una cultura organizacional.

Las instituciones públicas y privadas reconocen la importancia de fomentar el emprendimiento desde el sistema educativo superior; sin embargo, las acciones que se vienen realizando no están articuladas a fin de lograr los objetivos comunes. $\mathrm{Si}$ bien es cierto la mayoría de los estudiantes universitarios tienen los conocimientos técnicos para desarrollar un proyecto empresarial, estos mismos jóvenes no tienen el nivel motivacional necesario como para asegurar la inclinación por el emprendimiento como opción de realización personal y profesional.

A pesar de los esfuerzos realizados, la UNMSM debe replantear la formación de líderes emprendedores. Urge plantearnos esto como meta a corto plazo y contribuir, de esta manera, al desarrollo económico y social a nivel local, regional y nacional, a través de la creación de nuevas empresas.

Es fundamental fortalecer las redes de apoyo entre la universidad y las instituciones públicas y privadas que promuevan el emprendimiento, de este modo se podría suplir la carencia o desconocimiento respecto al tema promocional.

\section{CONCLUSIONES}

1. La mayoría de estudiantes de la Facultad de Ciencias Administrativas de la UNMSM, de las tres escuelas profesionales de Administración, Negocios Internacionales y Turismo, presentan puntajes que los clasifican según sus características en la categoría del nivel promedio (según 
los tres niveles de clasificaciones: Alto, Medio y Bajo) del Test del Emprendimiento diseñado por el Ministerio de Trabajo y Promoción del Empleo.

2. Se observó un mayor puntaje dentro de la categoría nivel promedio del Test de Emprendimiento de las siguientes características: Administra su tiempo, Tolerante y Líder.

3. La excepción de los resultados del nivel promedio de todas las características del Test de Emprendimiento tomadas en este universo de alumnos de la facultad de ciencias administrativas y que dieron como resultado un nivel bajo fueron en las dos siguientes características: Responsable y Confianza.

4. En cuanto a la distribución del nivel de emprendimiento entre las características del Test, se pudo apreciar lo siguiente:

- Existe porcentaje de alumnos con puntajes que lo ubican en la categoría del nivel alto en dos características: Visionario e Iniciativa.

- Se observa porcentajes de alumnos con puntuación que lo ubican en la categoría del nivel bajo en dos categorías: Superación y Confianza.

5. No se encuentra evidencia de diferencia significativa en la categoría de los puntajes de las características emprendedoras en las tres escuelas profesionales, salvo en la característica Optimista que en la escuela de Negocios Internacionales tiene la categoría promedio y en las otras escuelas tiene categoría baja.

6. Se puede apreciar que el nivel de emprendimiento entre las Escuelas Profesionales predomina el nivel promedio. No se aprecia diferencias significativas entre las carreras profesionales.

7. No existen diferencias significativas entre los niveles de estudio, salvo en las característica Optimista y Responsable, en los cuales los ciclos avanzados tienen un puntaje de categoría promedio, mientras que los ciclos iniciales tiene un puntaje en la categoría bajo. Se puede verificar en términos generales el comportamiento recurrente y homogéneo respecto a las características emprendedoras en las tres escuelas de la Facultad de Ciencias Administrativas de la Universidad Nacional Mayor de San Marcos.

\section{LITERATURA CITADA}

Alcarraz Rodríguez, Rafael. (2001). El emprendedor de éxito. Ed. Mc Rally, Mexico.

Assis Dornelas, José Carlos. (2001) Empreendedorismo. Transformando ideias en negocios. Mc Graw Hill, Brasil.

Bernal T., César Augusto. (2000). Metodología de la investigación para administración y economía. Pearson Educación de Colombia, Colombia.

Freyre, Andy. (2004). Pasión por emprender. Ed. Aguilar, Madrid.

Hernández, Roberto; Fernández, Carlos y Baptista, Pilar. (1991). Metodología de la investigación. Segunda edición. Mc Graw Hill, México.

Irigoyen, Horacio. (1997). PYMEs, su economía y organización. Mc Graw Hill, Brazil.

Jaramillo, Miguel y Parodi, Sandro. (2003). Jóvenes emprendedores. Apoyo, Lima.

Kushell, Jennifer (2001). Sólo para emprendedores, aproveche su juventud ambición e independencia para crear un negocio exitoso. Ed. Norma, Colombia.

Moreano León, Juan Antonio. (2005). El perfil psicosocial del emprendedor. Consejo Económico Social (CES), Madrid.

Moriano, Juan A. (2005). "Un estudio descriptivo sobre los emprendedores en España, la República Checa y Bulgaria”. En: Roig, Salvador; Ribeiro, Domingo; Torcal, V. Ramón; de la Torre, Amparo y Cerver, Elvira. (2004). El emprendedor innovador y la creación de empresas de I + D + I. I Congreso Internacional MOTIVA. Universitat de València, València. Fecha de consulta: 02/03/2013. Disponible en: < http://www.uv.es/motiva/ libromotiva/10MorianoSanchezPalaci.pdf $>$.

Sánchez C., Hugo y Reyes M., Carlos. (1998). Metodología y diseños de la investigación científica. Ed. Aguilar, Lima.

Sánchez Yábar, Guido y Becerra, S.F. (2005). Aprendiendo a emprender. Artes y Diseños Láser, Lima.

Serida, Jaime; Borda, Armando; Nakamatsu, Keiko; Morales, Oswaldo y Yamakawa, Meter. (2006). Global Entrepreneurship Monitor 2004-2005. ESAN Ediciones, Perú. 
Sofield, Mathew C. y Lusser, Robert. (1998). "El arte de emprender, cómo valorar las alternativas estratégicas del nuevo negocio". En: Harvard Deusto Business Review. Vol. 16, Nº2. Febrero de 1998.

Vinante, José Luis. (2004). "Emprendedor, creatividad, resultados". En: Iniciativas.net. En línea]. Fecha de consulta: 02/03/2013. Disponible en: < http://www.iniciativasnet. com/03.htm\# > .

\section{Páginas Web}

- www.uv.es/motiva/libromotiva/10MorianoSanc hezPalaci.pdf. EMPRENDEDORES EN ESPAÑA,. LA REPÚBLICA CHECA Y BULGARIA. Juan A. Moriano ... del estudio de los emprendedores. De Juan Moriano: jmoriano@bec.uned.es. 2005.

- www.iniciativasnet.com. EMPRENDEDOR. Creatividad - Reultados. Luis José Vinante. 2004. 